\title{
Slit1b-Robo3 Signaling and N-Cadherin Regulate Apical Process Retraction in Developing Retinal Ganglion Cells
}

\author{
Grace K. W. Wong, ${ }^{1}$ Marie-Laure Baudet, ${ }^{1}$ Caren Norden, ${ }^{1,2}$ Louis Leung, ${ }^{1,2}$ and William A. Harris ${ }^{1}$ \\ ${ }^{1}$ Department of Physiology, Development and Neuroscience, University of Cambridge, Cambridge CB2 3DY, United Kingdom, and ${ }^{2}$ Max Planck Institute of \\ Molecular Cell Biology and Genetics, 01307 Dresden, Germany
}

When neurons exit the cell cycle after their terminal mitosis, they detach from the apical surface of the neuroepithelium. Despite the fact that this detachment is crucial for further neurogenesis and neuronal migration, the underlying mechanisms are still not understood. Here, taking advantage of the genetics and imaging possibilities of the zebrafish retina as a model system, we show by knockdown experiments that the guidance molecule Slit $1 \mathrm{~b}$ and its receptor Robo3 are required for apical retraction of retinal ganglion cells (RGCs). In contrast, $\mathrm{N}$-cadherin seems to be responsible for maintenance of apical attachment, as expression of dominant-negative $\mathrm{N}$-cadherin causes RGCs to lose apical attachments prematurely and rescues retraction in slit1b morphants. These results suggest that Slit-Robo signaling downregulates $\mathrm{N}$-cadherin activity to allow apical retraction in newly generated RGCs.

\section{Introduction}

Upon neurogenesis in the vertebrate CNS, progenitors undergo divisions at the apical surface of the neuroepithelium, which in the final round of division gives rise to postmitotic neurons (Götz and Huttner, 2005). These newly generated neurons often first adopt a neuroepithelial morphology but then quickly detach their process from the apical surface and migrate toward the basal side of the neuroepithelium (Nadarajah et al., 2001; Miyata et al., 2004). Recent studies in the mouse cortex disagree on whether integrin function (Loulier et al., 2009) or adherens junctions (Cappello et al., 2006; Imai et al., 2006; Loulier et al., 2009) regulate retraction of apical processes. It is also not known whether extrinsic signals regulate this event.

In a study focused on axon initiation in transgenically labeled ath5:gapGFP retinal ganglion cells (RGCs), Zolessi et al. (2006) made the unexpected observation that slit $1 b$ morphants showed inhibition of apical retraction. They used this finding to demonstrate that axonogenesis, which happened on schedule, did not depend on apical retraction. However, Zolessi et al. (2006) did

Received May 25, 2011; revised 0ct. 21, 2011; accepted 0ct. 25, 2011.

Author contributions: G.K.W.W., M.-L.B., C.N., L.L., and W.A.H. designed research; G.K.W.W. and M.-L.B. performed research; G.K.W.W., C.N., L.L., and W.A.H. analyzed data; G.K.W.W., C.N., and W.A.H. wrote the paper.

This work was supported by a Wellcome Trust Programme Grant (to W.A.H.) and Croucher Foundation Scholarship (to G.K.W.W. during her PhD work). Some of the data not shown in this paper can be found in G.K.W.W.'s 2011 dissertation "Apical Process Retraction upon Neurogensis in the Zebrafish Retina" filed in the University Library at Cambridge University. We thank Flavio Zolessi and Lucia Poggi for sharing the morpholinos, ath5 constructs, and transgenic fish lines. We are grateful for the gifts of the PEGFP-N1-ncadh $\Delta$ EC by Assistant Professor James Jontes, the pCS2FA-transposase by Associate Professor Brian Link, and the different Tol2 entry clones and other constructs by Associate Professor Katharine Lewis and Professor Chi-bin Chien. We would also like to thank Jeremy Skepper for support with confocal microscopy; Adrian McNabb, Kirsty Scott and Tomasz Dyl for taking care of our animal stocks; Patricia Jusuf, Owen Randlett, Helen Lynn and Georgina Stooke-Vaughan for technical assistance; and Michalis Agathocleous for statistical advice.

Correspondence should be addressed to William A. Harris, Department of Physiology, Development and Neuroscience, Anatomy Building, University of Cambridge, Downing Street, Cambridge CB2 3DY, United Kingdom. E-mail: harris@mole.bio.cam.ac.uk.

DOI:10.1523/JNEUROSCI.2596-11.2012

Copyright $\odot 2012$ the authors $\quad 0270-6474 / 12 / 320223-06 \$ 15.00 / 0$ not investigate the mechanism of retraction. Slit proteins act as repulsive guidance ligands for axonal growth cones expressing Robo receptors and it has been reported that activated Robo or addition of purified Slit can inhibit N-cadherin-mediated cell adhesion in chick retinal cell cultures (Rhee et al., 2002, 2007). In Drosophila, Slit-Robo signaling negatively regulates cadherinmediated adhesion on cardioblasts to enable proper lumen formation (Medioni et al., 2008; Santiago-Martínez et al., 2008). These results raised the possibility that a similar mechanism mediates the retraction of apical processes in the neuroepithelium. We found that Slit1B or Robo3 downregulation in transgenically labeled RGCs leads to inhibition of apical retraction. Expression of dominant-negative N-cadherin in RGCs results in the opposite phenotype-premature apical detachment. $\mathrm{N}$-cadherin dominant-negative overexpression rescues apical retraction in slit $1 b$ morphants. These results are consistent with a model in which Slit/Robo signaling downregulates N-cadherinbased adhesion allowing apical retraction.

\section{Materials and Methods}

Animals. Wild-type and transgenic zebrafish were bred and kept at $26.5^{\circ} \mathrm{C}$. Embryos obtained from natural mating were raised in $0.003 \%$ phenylthiourea at $28.5^{\circ} \mathrm{C}$ to prevent pigment formation. Transgenic lines [Tg(ath5:gapGFP) and $\operatorname{Tg}($ ath 5: gapRFP $)]$ were from Zolessi et al. (2006).

Constructs and antibodies. Constructs used for embryo injection: ath5: gapGFP (Zolessi et al., 2006), hsp70:mCherryCAAX, and hsp70:ncadh $\Delta E C$ $m$ Cherry.pDONR-ncadh $\triangle E C$ was subcloned from $p E G F P-N 1$-ncadh $\Delta E C$, a gift from Dr. James Jontes (Ohio State University, Columbus, $\mathrm{OH}$ ), using primers to add att sites at the $5^{\prime}$ and $3^{\prime}$ ends of the N-cadherin sequence. hsp70:mCherryCAAX and hsp70:ncadh $\Delta E C$ - $m$ Cherry constructs were made using the Tol2kit as published (Kwan et al., 2007). Entry clones, except $p D O N R-n c a d h \Delta E C$, were gifts from Drs. Kate Lewis (Syracuse University, Syracuse, NY) and Chi-bin Chien (University of Utah, Salt Lake City, UT).

Primary antibodies used were diluted in blocking solution as follows: Zn5, 1:500 (Zebrafish International Resource Center); anti-N-cadherin antibody, 1:200 (ab12221; Abcam); anti-GFP, 1:200 (11814460001; Roche); and anti-GFP-FITC-conjugated, 1:500 (ab6662; Abcam). Sec- 


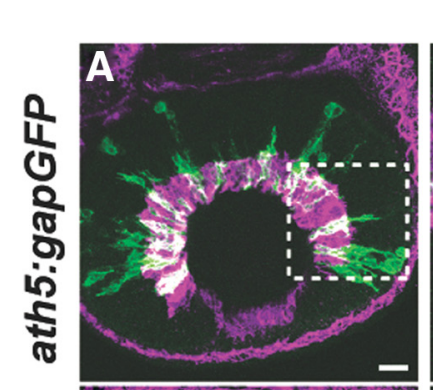

\section{Ath5:gapGFP Zn5}
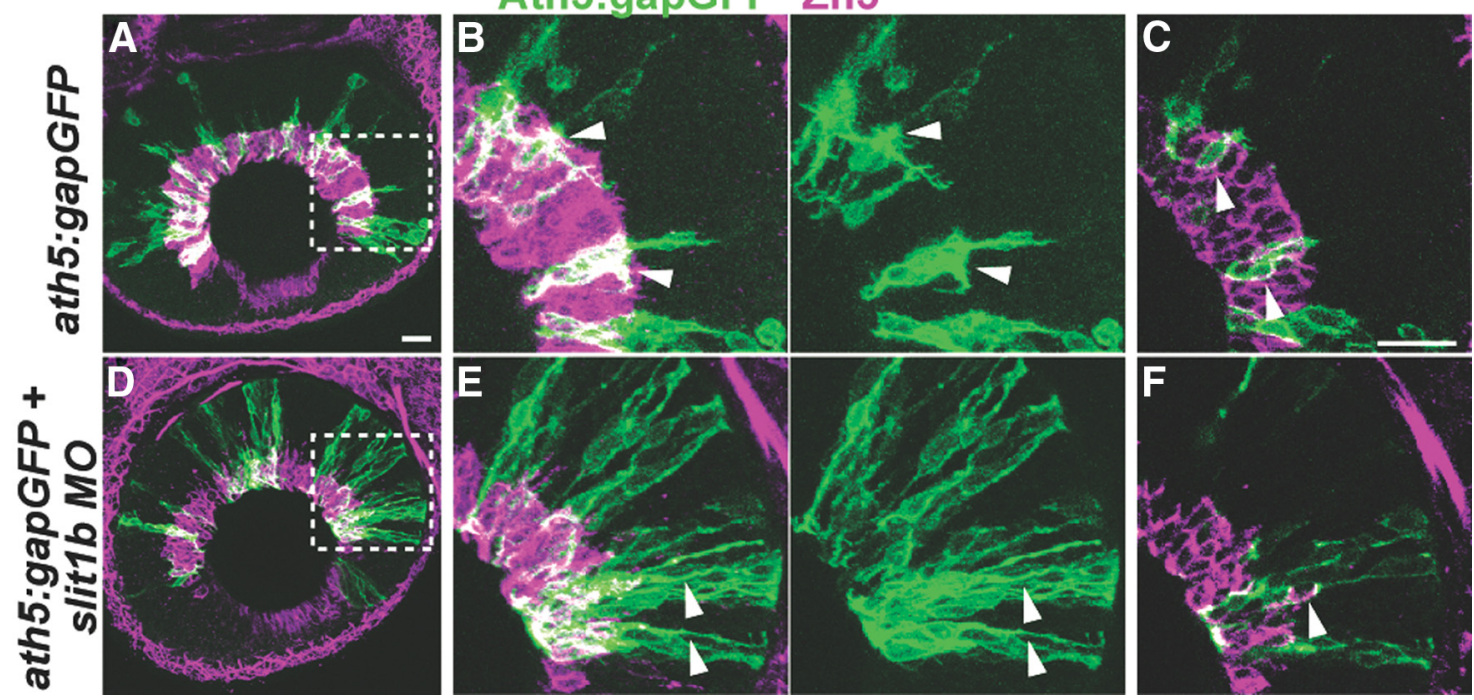

Figure 1. Apical processes retraction of RGCs is delayed in slit1 $b$ morphants. Analysis was performed on ath5:gap GFP DNA-injected embryos at 48 hpf. $A, D$, Extended-focus confocal images of the retinas of a control embryo $(\boldsymbol{A})$ or a slit1 $1 b$ morphant $(\boldsymbol{D})$. Boxed regions in $\boldsymbol{A}$ and $\boldsymbol{D}$ are enlarged in $\boldsymbol{B}$ and $\boldsymbol{E}$, respectively. Arrowheads point to the retracted apical processes in the control retina $(\boldsymbol{B})$ and un-retracted apical processes in the slit7b morphant retina (E). Zn5 (magenta) labels the ganglion cell soma and axon. $\boldsymbol{C}, \boldsymbol{F}$, Single optical section images of the boxed regions in $\boldsymbol{A}$ and $\boldsymbol{D}$, respectively, showing that the Ath5:gapGFP + cells express Zn5 and are thus RGCs (arrowheads). Scale bars, $20 \mu \mathrm{m}$.

ondary antibodies used were as follows: donkey or goat anti-mouse IgG conjugated to Alexa 488, 594, or 647 fluorophores (Invitrogen), diluted to $1: 1000$ in blocking solution.

Image analyses. Optical sections of whole-mount embryos were obtained with a confocal microscope (SP2; Leica) at $\sim 0.6 \mu \mathrm{m}$ intervals, as described previously (Poggi et al., 2005). Images were analyzed using Volocity software (Improvision). Using Zn5 (or ath5:gapGFP; see Fig. 3) as a marker for the differentiated ganglion cells, a differentiated RGC with retracted apical process had both its soma and apical process located within the labeled ganglion cell layer, while a RGC with an un-retracted apical process was defined as one with a positive cell body that retained an apical process outside of the ganglion cell layer. Counting was performed manually.

DNA, RNA, and morpholinos injections. Plasmid DNA encoding the fluorescent proteins were injected into the cytoplasm of one-cell stage zebrafish embryos. ath5:gapGFP construct was injected together with meganuclease $I-S c e I$ at a concentration of $10 \mathrm{ng} / \mu \mathrm{l}$ (Zolessi et al., 2006). To improve integration and expression of the transgenes, all the Tol2 constructs were injected with the transposase RNA in a 1:1 ratio (Kwan et al., 2007). RNA and morpholinos (MOs; Genetools) were injected into the yolk of one- to four-cell stage embryos. MOs used were as follows: standard control MO (5'-CCTCTTACCTCAGTTACAATTTATA- $\left.3^{\prime}\right)$; 0.2 ng of anti-slit $1 b$, translation blocking (5'-GCTCGGTGTCCGGCAT CTCCAAAAG-3') (Zolessi et al., 2006); 2 ng of anti-robo2, translation blocking (5'-GTAAAAGGTGTGTTAAAGGACCCAT-3'); and a combination of $1 \mathrm{ng}$ of anti-robo3varl, translation blocking (5'-CCCTAAAA GCGCTACAATCCACCTG-3' ${ }^{\prime}$ ) and 1 ng of anti-robo3var2, translation blocking ( $5^{\prime}$-TCTTTATCAGGAACGCAGCATCTC-3') (Challa et al., 2005; Devine and Key, 2008).

Blastomere transplantation. Embryos were dechorionated with Pronase $(30 \mathrm{mg} / \mathrm{ml}$; Sigma). Blastomeres from transgenic donor embryos, $\operatorname{Tg}$ (ath5:gapRFP), injected with $H 2 B-Y F P$ mRNA, and morpholinos were transplanted into the animal poles of unlabeled host blastulas using a glass micropipette.

Heat shock experiments. Embryos injected with the heat-shock promoter-driven constructs were raised at $28.5^{\circ} \mathrm{C}$ until 20 or $24 \mathrm{~h}$ postfertilization (hpf). The embryos were then incubated at room temperature for 30-60 min, transferred to a tube of prewarmed medium, and heat-shocked at $37^{\circ} \mathrm{C}$ for $1 \mathrm{~h}$.

RNA synthesis. pCS2FA-transposase was a gift from Dr. Brian Link (Medical College of Wisconsin, Milwaukee, WI). The transposase RNA was transcribed using the mMessage machine in vitro transcription kit (Ambion) from the SP6 promoter of $p C S 2 F A$-transposase, after linear- ization with NotI. The mRNA was subsequently purified using RNeasy RNA purification kit (Qiagen).

In situ hybridization. slit $1 b$ and robo3 antisense probes were generated by digesting $p C R 2.1-T O P O-s l i t 1 b-2 C$ and pCR2.1-TOPO-robo3 with BamH1 and HindIII, respectively, then transcribing with T7 RNA polymerase. pCR2.1-TOPO-slit1b-2C and pCR2.1-TOPO-robo3 were gifts from Dr. Chi-bin Chien. Whole-mount in situ hybridization of slit1b mRNA was performed on wild-type embryos as previously described (Shimamura et al., 1994), hybridized embryos were subsequently sectioned for image acquisition. In situ hybridization for robo 3 mRNA was performed on $20 \mu \mathrm{m}$ cryosections as previously described (Butler et al., 2001).

Statistical analysis. The Mann-Whitney $U$ test was used to compare the percentage of Ath5:gapGFP-expressing cells with unretracted apical processes in WT and slit1b morphants per retina, using InStat software (GraphPad). Binomial test was used in all other experiments to assess statistical significance.

\section{Results}

\section{Slit1b and Robo3 regulate apical process retraction of RGCs}

To validate and extend the findings of Zolessi et al. (2006) on the role of Slit1b in apical retraction, embryos were injected with ath5:gapGFP with or without slit $1 b$ morpholino and fixed at 48 hpf. As Ath5:gapGFP is expressed by both the differentiated RGCs and their progenitors, Zn5, which labels the axon and soma of RGCs in zebrafish, was used as a definitive marker for the differentiated ganglion cells (Schmitt and Dowling, 1996). An Ath5:gapGFP + RGC was judged to have an un-retracted apical process if its cell body was in the RGC layer and positive for Zn5 yet retained an apical process that extended outside of the ganglion cell layer (Fig. 1 E, F, arrowheads). At 48 hpf, only few RGCs in control retinas had un-retracted apical processes, while a significant number of RGCs in the slit $1 b$ morphant retina retained un-retracted apical processes (Fig. 1). The reason that the RGC layer is thinner in slit $1 b$ morphants is probably that many RGC somas have trouble migrating basally when still attached apically (Zolessi et al., 2006). To check that this phenotype is not a result of a general developmental delay, we compared the relative timing of apical retraction to another RGC developmental event - axonogenesis using time-lapse analysis of Ath5:gapGFP-expressing RGCs 

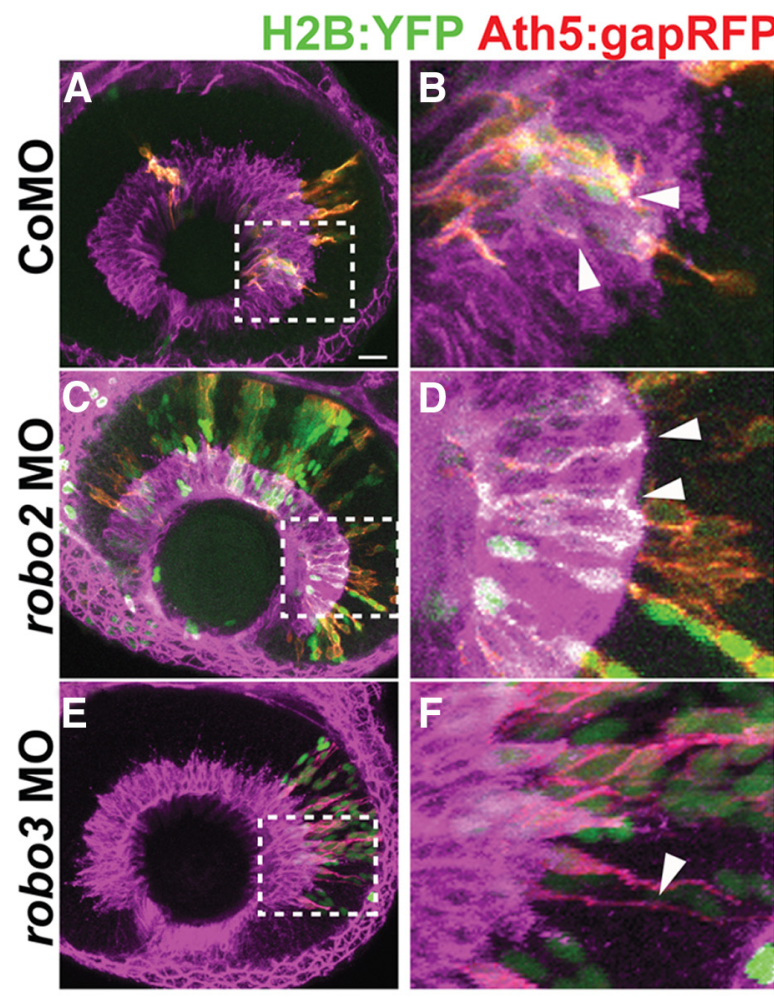

\section{Zn5}

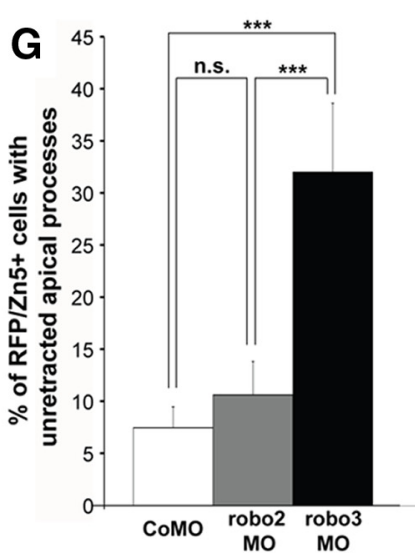

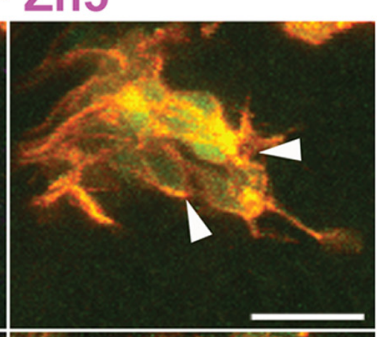
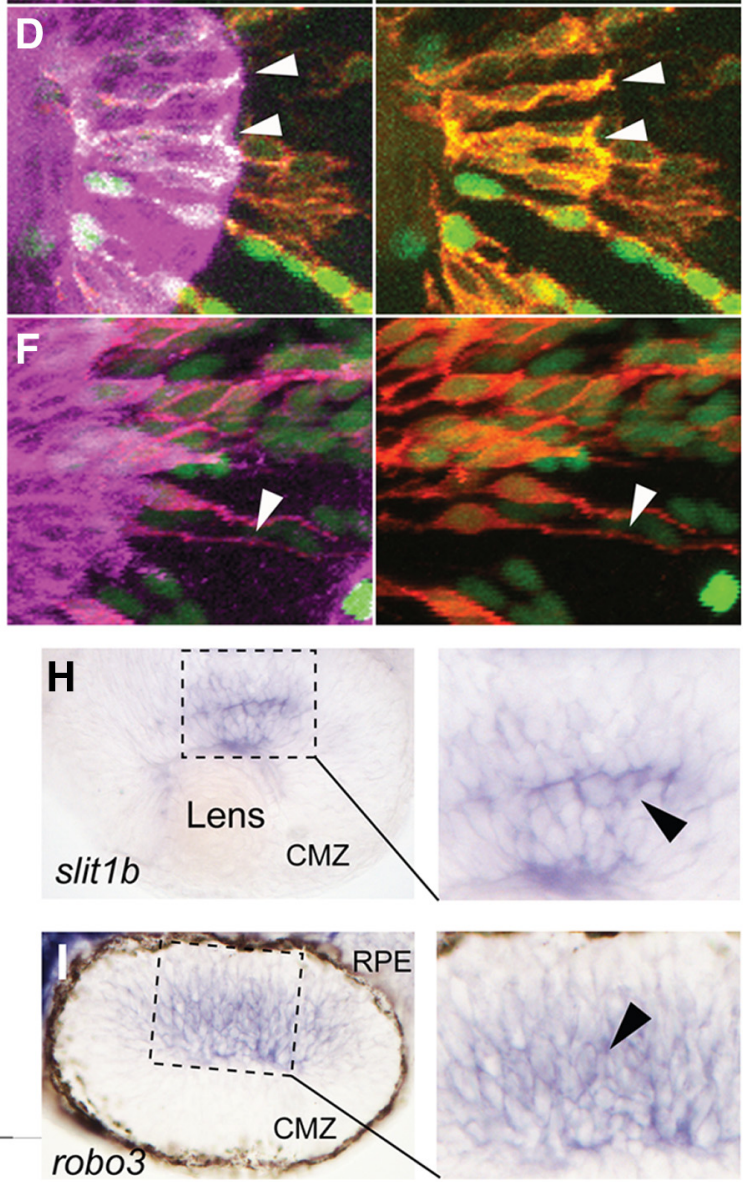

mediate the Slit1b-regulated apical retraction. Among the four zebrafish robo genes, only robo 2 and robo3 mRNA are detected in the developing retina (Lee et al., 2001). In zebrafish, two forms of robo3 exist: robo3v1 and robo3v2 (Challa et al., 2005). Therefore, to test whether Robo 2 or 3 in RGCs is involved in apical process retraction, we injected $\operatorname{Tg}($ ath5:gapRFP) embryos with control, robo2, or a combination of robo $3 v 1$ and robo $3 v 2$ morpholinos and $H 2 B-Y F P$ mRNA as a nuclear marker of donor cells. Blastomeres from injected embryos were then transplanted into uninjected wild-type hosts of the same stage. We found that Ath5: gapRFP-positive cells from control and robo 2 morpholino-injected embryos retracted their apical processes normally, while Ath5:gapRFP-positive cells from robo3 morphants retained apical processes (Fig. $2 B, D, F$ ). Thus, robo3 knockdown, but not robo2 knockdown, phenocopies slit1b morphants, suggesting that Robo3 is the receptor downstream of Slit1b during apical retraction.

An important question is why Slit $1 b$ and Robo3 do not cause neuroepithelial cells to detach before their final division. RGCs of the embryonic zebrafish retina go through their final S-phase between 30 and $40 \mathrm{hpf}$ (Hu and Easter, 1999) and finish mitosis a few hours later (Poggi et al., 2005). Using in situ hybridization at a variety of time points between 24 and $40 \mathrm{hpf}$, we first detected a clear signal for both slit $1 b$ and robo 3 mRNA in scattered cells at 236-38 hpf (Fig. $2 H, I$ ). Thus, the timing of expression of this receptor-ligand pair may provide part of the answer to this question.

Figure 2. Knockdown of robo3 but not robo2 inhibits apical process retraction. $\boldsymbol{A}-\boldsymbol{F}$, Confocal images from wild-type hosts transplanted with blastomeres from $\operatorname{Tg}($ ath5:gapRFP) embryos injected with control morpholino (CoM0;A, B), robo2 morpholino $(\boldsymbol{C}, \boldsymbol{D})$, or a combination of robo3v1 and $v 2$ morpholinos $(\boldsymbol{E}, \boldsymbol{F})$ at $48 \mathrm{hpf}$. $Z \mathrm{n} 5$ (magenta) labels the ganglion cell layer. Boxed regions in $\boldsymbol{A}, \boldsymbol{C}$, and $\boldsymbol{E}$ are enlarged in $\boldsymbol{B}, \boldsymbol{D}$, and $\boldsymbol{F}$, respectively. Arrowheads in $\boldsymbol{B}$ and $\boldsymbol{D}$ indicate the retracted apical processes of transplanted Ath5:gapRFP+ RGCs from control or robo2 morpholinos-injected embryos; arrowheads in $\boldsymbol{F}$ indicate the un-retracted apical process of a transplanted RGC from a robo3v1 and $v 2$ double-morphant. Scale bars, $20 \mu \mathrm{m}$. G, Percentage of Ath5:gapRFP and Zn5 double-positive cells with un-retracted apical processes at $48 \mathrm{hpf}(n=187 \mathrm{cells}$ from control morpholino-injected embryos; $n=$ 94 cells from robo 2 morphants; $n=50$ cells from robo3 morphants). Error bars indicate SEs (*** $p<0.001) . \boldsymbol{H}, \boldsymbol{I}$, A retinal section from a $38 \mathrm{hpf}$ whole-mount in situ hybridized embryo, showing that slit $1 b$ and robo3 mRNAs are expressed in the developing zebrafish retina. I, In situ hybridization image from a similarly staged retinal section showing that robo3 mRNA is expressed in subsets of cells in the developing retina. Note that pigments were allowed to form and the retinal pigmented epithelium (RPE) is thus visible. CMZ, Ciliary marginal zone.

starting at $35 \mathrm{hpf}$. We found that only $31.6 \pm 10.6 \%$ of normal RGCs sent out axons before retracting their apical processes $(n=19)$, but in slit1b morphants, although axonogenesis was not premature, $68.4 \pm 9.1 \%$ of RGCs extended an axon while retaining an apical process $(n=26)$. This significant difference $(p<0.01)$ indicates that apical retraction is inhibited compared with axonogenesis by the knocking down Slit1b.

Robo proteins are receptors for Slit ligands. Therefore, we wondered whether newborn RGCs express Robo, which could

\section{Expression of dominant-negative} $\mathrm{N}$-cadherin leads to premature apical process retraction

Immunohistochemistry reveals that $\mathrm{N}$ cadherin is expressed strongly in the developing zebrafish retina (Liu et al., 2001), consistent with a possible role in apical retraction. As $\mathrm{N}$-cadherin is expressed in the apical processes of Ath5:gapGFPpositive RGC progenitors (Fig. $3 A$ ), we investigated whether it is involved in apical process attachment using a dominantnegative construct lacking the extracellular $\mathrm{N}$-cadherin domain $(\mathrm{Ni}$ eman et al., 1999; Jontes et al., 2004). To bypass the severe defects that result from disrupting $\mathrm{N}$-cadherin in early development (Pujic and Malicki, 2001), we controlled the expression of the dominantnegative protein by a heat-shock-inducible promoter. Expression was induced by a $1 \mathrm{~h}$ heat-shock at $37^{\circ} \mathrm{C}$ at $20 \mathrm{hpf}$ in $\operatorname{Tg}(a t h 5$ : gapGFP) embryos either injected with $h s p 70: n c a d h \Delta E C$-mCherry or a control construct ( $h s p 70: m C h e r r y C A A X)$. The injected embryos were fixed at $36 \mathrm{hpf}$. As most RGCs have just exited cell cycle at 36 
hpf, we speculated that most of the newly born RGCs would still retain apical processes (Hu and Easter, 1999). As expected, the majority of the Ath5:gapGFP + cells expressing hsp70:mCherryCAAX had unretracted processes attached to the apical surface. However, Ath5:gapGFP+ cells expressing the dominant-negative $\mathrm{N}$-cadherin protein had fewer un-retracted apical process (Fig. 3D). Thus, N-cadherin function appears to be essential for the attachment of newborn RGC apical processes.

Dominant-negative $\mathrm{N}$-cadherin rescues the retraction phenotype in slit1b morphants

As we found that blocking Slit1b/Robo3 signaling inhibits apical retraction, whereas interfering with $\mathrm{N}$-cadherin function leads to precocious retraction in developing RGCs. One possible explanation for these opposing phenotypes is that Slit/ Robo signaling normally downregulates $\mathrm{N}$-cadherin-mediated apical adhesion. If this was the case, the dominant-negative $\mathrm{N}$-cadherin phenotype should be epistatic to the Slit1b phenotype. To test this notion, embryos were coinjected with control morpholino and hsp70:mCherryCAAX or hsp70:ncadh $\Delta E C-m$ Cherry, or with slit1b morpholino and hsp70:mCherryCAAX or hsp70:ncadh $\Delta E C$-mCherry. Embryos were then heat-shocked at $24 \mathrm{hpf}$ and fixed at 48 hpf. Knockdown of Slit1b was confirmed to inhibit apical retraction, as shown by a significantly higher proportion of Hsp70: mCherryCAAX RGCs with un-retracted apical processes in slit $1 b$ morphants compared with those in the control morphants (Fig. 4A-F). The Hsp70:Ncadh $\Delta$ ECmCherry-expressing RGCs, however, retracted their apical processes efficiently in these morphants (Fig. 4G), showing that blocking $\mathrm{N}$-cadherin function in the morphant cells rescues apical retraction. These results suggest a model in which Slit-Robo signaling in newborn RGCs downregulates $\mathrm{N}$-cadherin-mediated attachment at the apically located adherens junction, allowing apical retraction upon cell cycle exit.

\section{Discussion}

In this report, we define a putative molecular pathway underlying the retraction of apical processes in newborn RGCs. We first show that knocking down Slit1b inhibits apical process retraction, corroborating previous data (Zolessi et al., 2006). We identified Robo3 as the likely receptor for Slit1b in this process. As work in other systems has shown that Slit/Robo signaling is capable of modulating cadherin function (Rhee et al., 2002; Medioni et al., 2008; Santiago-Martínez et al., 2008), we next investigated whether $\mathrm{N}$-cadherin, which is known to be part of the adherens junctions at the apical complex in neuroepithelial cells, is also involved in the process of RGC apical retraction. We
N-cad Ath5:gapGFP
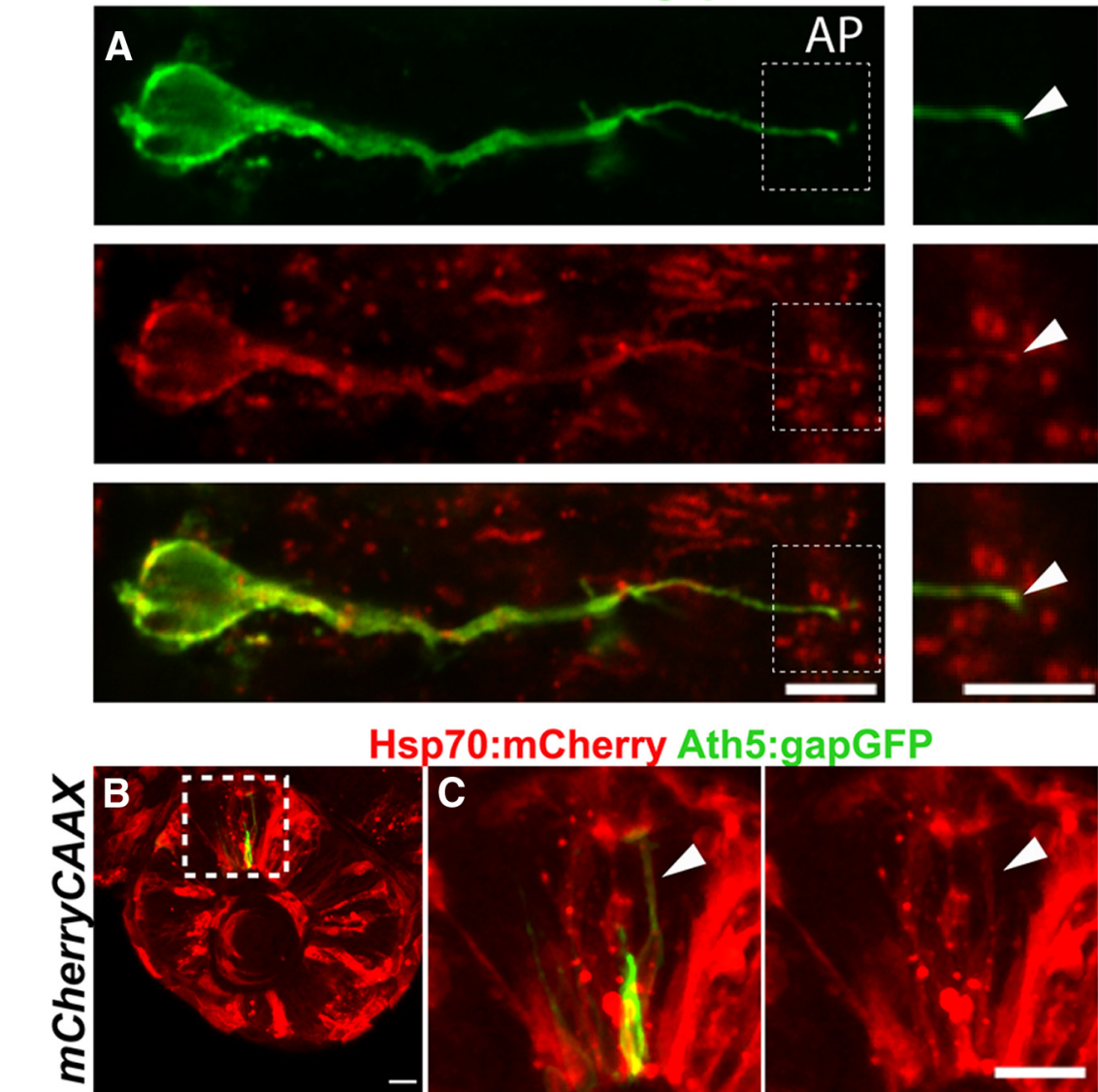

sp70:mCherry Ath5:gapGFP
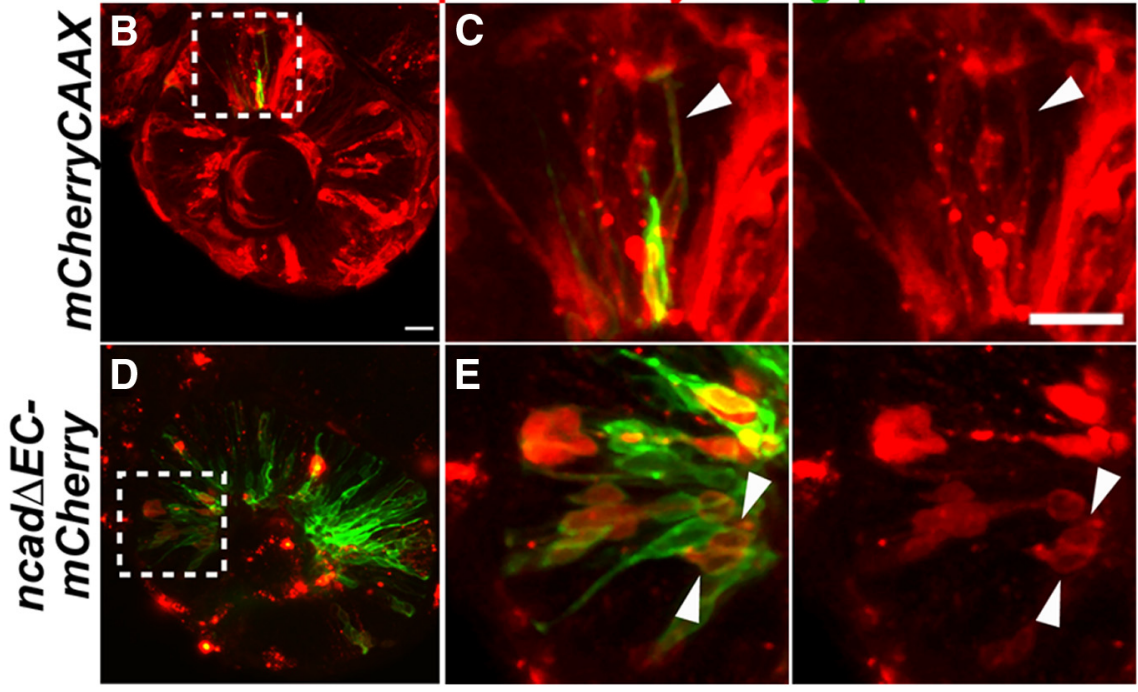

Figure 3. Expression of heat-shock-induced dominant-negative $\mathrm{N}$-cadherin leads to premature apical processes retraction in RGC progenitors. $\boldsymbol{A}$, Confocal images of the apical region (AP) of the retina of a 36hpf $\operatorname{Tg}($ ath5:gapGFP) embryo stained with (arrowheads). $\boldsymbol{B}-\boldsymbol{E}$, Confocal images of the retinas of $\operatorname{Tg}($ ath5:gapGFP) embryos injected with hsp70:mCherry $(A A X(\boldsymbol{B}, \boldsymbol{C})$ or hsp70:ncadh $\triangle E C-m C h e r r y(\boldsymbol{D}, \boldsymbol{E})$ at $36 \mathrm{hpf}$. Boxed regions in $\boldsymbol{B}$ and $\boldsymbol{D}$ are enlarged in $\boldsymbol{C}$ and $\boldsymbol{E}$, respectively. Arrowheads in $\boldsymbol{C}$ indicate the un-retracted apical process of an Ath5:gapGFP-positive cell expressing Hsp70:mCherryCAAX. Arrowheads in $\boldsymbol{E}$ indicate Ath5: gapGFP-positive cells expressing Hsp70:Ncadh $\Delta$ EC-mCherry with retracted apical processes. Scale bars, $20 \mu \mathrm{m}$.

found that the expression of a dominant-negative $\mathrm{N}$-cadherin in RGCs leads to premature detachment of their apical processes and rescues the apical detachment phenotype in slit $1 b$ morphants.

Our results are thus consistent with the possibility that $\mathrm{N}$-cadherin is downregulated by Slit-Robo signaling, leading to the loss of apical adhesion. To establish further details of this molecular pathway, it will be necessary to investigate the links between Slit/Robo signaling and N-cadherin. Epithelial/mesenchyme transition has been found to be mediated by Rab5dependent endocytosis of E-cadherin (Fujita et al., 2002; Palacios et al., 2005; Emery and Knoblich, 2006), and E-cadherin internalization can be induced by soluble factors (Kamei et al., 1999; Ulrich et al., 2005; Bryant et al., 2007). Preliminary experiments 


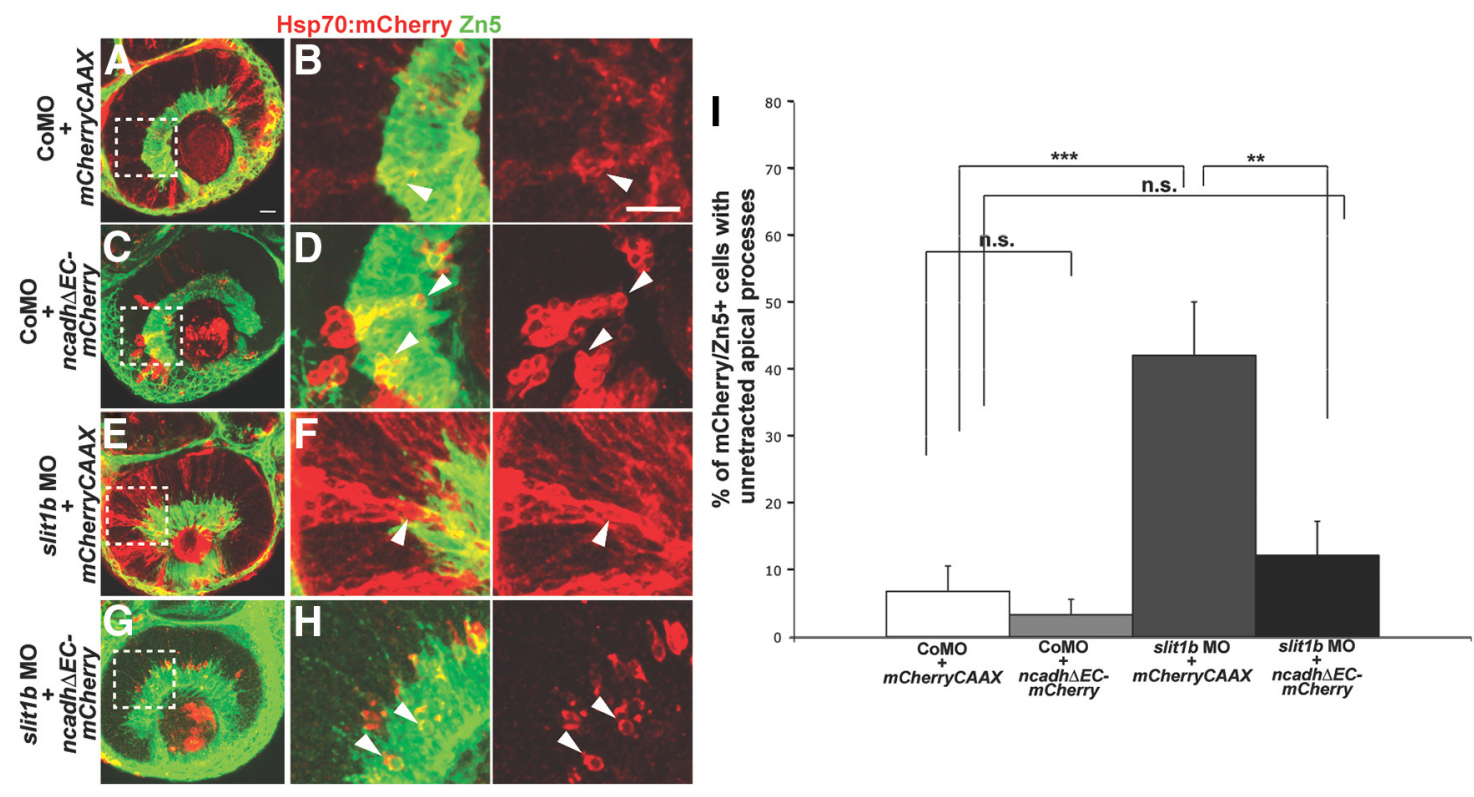

Figure 4. Expression of dominant-negative N-cadherin rescues apical retraction in slit1b morphant RGCs. $\boldsymbol{A}-\boldsymbol{H}$, Confocal images from control morpholino-injected (CoMO) embryos expressing Hsp70:mCherryCAAX $(\boldsymbol{A}, \boldsymbol{B})$ or Hsp70:Ncadh $\triangle \mathrm{EC}-\mathrm{mCherry}(\boldsymbol{C}, \boldsymbol{D})$; confocal images from slit7b morphants expressing Hsp70:mCherryCAAX $(\boldsymbol{E}, \boldsymbol{F})$ or Hsp70:Ncadh $\Delta \mathrm{EC}-\mathrm{mCherry}(\boldsymbol{G}, \boldsymbol{H})$ at 48 hpf. Zn5 (pseudocolored in green) labels the ganglion cells. Boxed regions in $\boldsymbol{A}, \boldsymbol{C}, \boldsymbol{E}$, and $\boldsymbol{G}$ are enlarged in $\boldsymbol{B}, \boldsymbol{D}, \boldsymbol{F}$, and $\boldsymbol{H}$, respectively. Arrowheads in $\boldsymbol{F}$ indicate the un-retracted apical process of an Hsp70:mCherryCAAX-expressing RGC (defined as cells that are Zn5 +) in a slit7b morphant; arrowheads in $\boldsymbol{B}, \boldsymbol{F}$, and $\boldsymbol{H}$ indicate mCherry-positive RGCs with retracted apical processes. Scale bars, 20 $\mu \mathrm{m}$. I, Percentage of mCherry-positive RGCs with un-retracted apical processes at $48 \mathrm{hpf}(n=44 \mathrm{Hsp} 70$ :mCherryCAAX-expressing cells from control morpholino-injected embryos; $n=61$ Hsp70:Ncadh $\Delta$ EC-mCherry-expressing cells from control morpholino-injected embryos; $n=38 \mathrm{Hsp} 70$ :mCherryCAAX-expressing cells from slit1b morphants; $n=41 \mathrm{Hsp} 70: \mathrm{Ncadh} \Delta \mathrm{EC}-\mathrm{mCherry-}$ expressing cells from slit1 16 morphants). Error bars indicate SEs $\left({ }^{* *} p<0.01 ;{ }^{* * *} p<0.001\right)$.

with endocytosis inhibitors and the expression of dominantnegative Rab5, however, failed to interfere with apical retraction of RGCs in our system (data not shown), suggesting that other effectors might be at play. Likely candidates include those discovered in cell culture systems by Rhee et al. $(2002,2007)$, who found that Slit activation of Robo leads to the phosphorylation of $\beta$-catenin by Abl; this causes a decrease in the binding affinity of $\beta$-catenin for $\mathrm{N}$-cadherin and reduces $\mathrm{N}$-cadherin-mediated adhesion by severing its link to the cytoskeleton.

Transient disruption of integrin functions by injection of blocking antibody in mouse ventricular zone leads to detachment of apical processes from the ventricular surface (Loulier et al., 2009); this suggests that integrin signaling could also play a role in the apical attachment of neuroepithelial cells in the developing mouse cortex. Our results, though more consistent with a role for adherens junctions in apical attachment as suggested by Cappello et al. (2006) and Imai et al. (2006), do not rule out the possibility that both cadherins and integrins are involved in the attachments of apical processes, and that neither of them alone is sufficient. Given the expression of Slits, Robos, and Cadherin in different developing tissues (Bitzur et al., 1994; Challa et al., 2001; Lee et al., 2001; Hutson et al., 2003), we propose that Slit/Robo signaling and cadherin downregulation may be a common mechanism of apical processes retraction.

Precocious detachment affects the proliferation potential (Cappello et al., 2006; Imai et al., 2006), and failure to retract would inhibit the temporally orchestrated migration to distant sites, such as the cerebral cortex. Obviously a key issue for future research is what regulates the timing of this signaling. One attractive possibility in the retina is that Robo3 is only expressed when cells have completed their final mitosis. Our in situ results on the timing and localized expression of robo3 mRNA in subsets of retinal cells are consistent with this idea. Interestingly, Slit1b expression is also turned on just at this stage, so, even if retinal cells prematurely express Robo3, they cannot detach until Slit1b is expressed and vice versa. We hope this work will stimulate further research into these relatively unexplored questions of developmental cell biology.

\section{References}

Bitzur S, Kam Z, Geiger B (1994) Structure and distribution of N-cadherin in developing zebrafish embryos: morphogenetic effects of ectopic overexpression. Dev Dyn 201:121-136.

Butler K, Zorn AM, Gurdon JB (2001) Nonradioactive in situ hybridization to Xenopus tissue sections. Methods 23:303-312.

Bryant DM, Kerr MC, Hammond LA, Joseph SR, Mostov KE, Teasdale RD, Stow JL (2007) EGF induces macropinocytosis and SNX1-modulated recycling of E-cadherin. J Cell Sci 120:1818-1828.

Cappello S, Attardo A, Wu X, Iwasato T, Itohara S, Wilsch-Bräuninger M, Eilken HM, Rieger MA, Schroeder TT, Huttner WB, Brakebusch C, Götz M (2006) The rho-GTPase cdc42 regulates neural progenitor fate at the apical surface. Nat Neurosci 9:1099-1107.

Challa AK, Beattie CE, Seeger MA (2001) Identification and characterization of roundabout orthologs in zebrafish. Mech Dev 101:249-253.

Challa AK, McWhorter ML, Wang C, Seeger MA, Beattie CE (2005) Robo3 isoforms have distinct roles during zebrafish development. Mech Dev 122:1073-1086.

Devine CA, Key B (2008) Robo-Slit interactions regulate longitudinal axon pathfinding in the embryonic vertebrate brain. Dev Biol 313:371-383.

Emery G, Knoblich JA (2006) Endosome dynamics during development. Curr Opin Cell Biol 18:407-415.

Fujita Y, Krause G, Scheffner M, Zechner D, Leddy HE, Behrens J, Sommer T, Birchmeier W (2002) Hakai, a c-Cbl-like protein, ubiquitinates and induces endocytosis of the E-cadherin complex. Nat Cell Biol 4:222-231.

Götz M, Huttner WB (2005) The cell biology of neurogenesis. Nat Rev Mol Cell Biol 6:777-788.

Hu M, Easter SS (1999) Retinal neurogenesis: the formation of the initial central patch of postmitotic cells. Dev Biol 207:309-321.

Hutson LD, Jurynec MJ, Yeo SY, Okamoto H, Chien CB (2003) Two divergent slitl genes in zebrafish. Dev Dyn 228:358-369.

Imai F, Hirai S, Akimoto K, Koyama H, Miyata T, Ogawa M, Noguchi S, Sasaoka T, Noda T, Ohno S (2006) Inactivation of aPKClambda results 
in the loss of adherens junctions in neuroepithelial cells without affecting neurogenesis in mouse neocortex. Development 133:1735-1744.

Jontes JD, Emond MR, Smith SJ (2004) In vivo trafficking and targeting of $\mathrm{N}$-cadherin to nascent presynaptic terminals. J Neurosci 24:9027-9034.

Kamei T, Matozaki T, Sakisaka T, Kodama A, Yokoyama S, Peng YF, Nakano K, Takaishi K, Takai Y (1999) Coendocytosis of cadherin and c-Met coupled to disruption of cell-cell adhesion in MDCK cells-regulation by Rho, Rac and Rab small G proteins. Oncogene 18:6776-6784.

Kwan KM, Fujimoto E, Grabher C, Mangum BD, Hardy ME, Campbell DS, Parant JM, Yost HJ, Kanki JP, Chien CB (2007) The Tol2kit: a multisite gateway-based construction kit for Tol2 transposon transgenesis constructs. Dev Dyn 236:3088-3099.

Lee JS, Ray R, Chien CB (2001) Cloning and expression of three zebrafish roundabout homologs suggest roles in axon guidance and cell migration. Dev Dyn 221:216-230.

Liu Q, Babb SG, Novince ZM, Doedens AL, Marrs J, Raymond PA (2001) Differential expression of cadherin-2 and cadherin-4 in the developing and adult zebrafish visual system. Vis Neurosci 18:923-933.

Loulier K, Lathia JD, Marthiens V, Relucio J, Mughal MR, Tang SC, Coksaygan T, Hall PE, Chigurupati S, Patton B, Colognato H, Rao MS, Mattson MP, Haydar TF, Ffrench-Constant C (2009) betal integrin maintains integrity of the embryonic neocortical stem cell niche. PLoS Biol 7:e1000176.

Medioni C, Astier M, Zmojdzian M, Jagla K, Sémériva M (2008) Genetic control of cell morphogenesis during Drosophila melanogaster cardiac tube formation. J Cell Biol 182:249-261.

Miyata T, Kawaguchi A, Saito K, Kawano M, Muto T, Ogawa M (2004) Asymmetric production of surface-dividing and non-surface-dividing cortical progenitor cells. Development 131:3133-3145.

Nadarajah B, Brunstrom JE, Grutzendler J, Wong RO, Pearlman AL (2001) Two modes of radial migration in early development of the cerebral cortex. Nat Neurosci 4:143-150.

Nieman MT, Kim JB, Johnson KR, Wheelock MJ (1999) Mechanism of extracellular domain-deleted dominant negative cadherins. J Cell Sci 112:1621-1632.
Palacios F, Tushir JS, Fujita Y, D’Souza-Schorey C (2005) Lysosomal targeting of E-cadherin: a unique mechanism for the down-regulation of cellcell adhesion during epithelial to mesenchymal transitions. Mol Cell Biol 25:389-402.

Poggi L, Vitorino M, Masai I, Harris WA (2005) Influences on neural lineage and mode of division in the zebrafish retina in vivo. J Cell Biol 171:991-999.

Pujic Z, Malicki J (2001) Mutation of the zebrafish glass onion locus causes early cell-nonautonomous loss of neuroepithelial integrity followed by severe neuronal patterning defects in the retina. Dev Biol 234:454-469.

Rhee J, Mahfooz NS, Arregui C, Lilien J, Balsamo J, VanBerkum MF (2002) Activation of the repulsive receptor Roundabout inhibits N-cadherinmediated cell adhesion. Nat Cell Biol 4:798-805.

Rhee J, Buchan T, Zukerberg L, Lilien J, Balsamo J (2007) Cables links Robo-bound Abl kinase to N-cadherin-bound beta-catenin to mediate Slit-induced modulation of adhesion and transcription. Nat Cell Biol 9:883-892.

Santiago-Martínez E, Soplop NH, Patel R, Kramer SG (2008) Repulsion by Slit and Roundabout prevents Shotgun/E-cadherin-mediated cell adhesion during Drosophila heart tube lumen formation. J Cell Biol 182:241-248.

Schmitt EA, Dowling JE (1996) Comparison of topographical patterns of ganglion and photoreceptor cell differentiation in the retina of the zebrafish, Danio rerio. J Comp Neurol 371:222-234.

Shimamura K, Hirano S, McMahon AP, Takeichi M (1994) Wnt-1dependent regulation of local E-cadherin and alpha $\mathrm{N}$-catenin expression in the embryonic mouse brain. Development 120:2225-2234.

Ulrich F, Krieg M, Schötz EM, Link V, Castanon I, Schnabel V, Taubenberger A, Mueller D, Puech PH, Heisenberg CP (2005) Wnt11 functions in gastrulation by controlling cell cohesion through Rab5c and E-cadherin. Dev Cell 9:555-564.

Zolessi FR, Poggi L, Wilkinson CJ, Chien CB, Harris WA (2006) Polarization and orientation of retinal ganglion cells in vivo. Neural Dev 1:2. 